\title{
Apprendre à enseigner dans le supérieur : quels modèles pour la construction des compétences des universitaires novices?
}

Sacha Kiffer

\section{(2) OpenEdition \\ Journals}

Édition électronique

URL : http://journals.openedition.org/ripes/1654

ISSN : 2076-8427

Éditeur

Association internationale de pédagogie universitaire

\section{Référence électronique}

Sacha Kiffer, "Apprendre à enseigner dans le supérieur : quels modèles pour la construction des compétences des universitaires novices ? ", Revue internationale de pédagogie de l'enseignement supérieur [En ligne], 34(3) | 2018, mis en ligne le 20 novembre 2018, consulté le 08 septembre 2020 URL : http://journals.openedition.org/ripes/1654 


\title{
Apprendre à enseigner dans le supérieur : quels modèles pour la construction des compétences des universitaires novices?
}

\author{
Sacha Kiffer
}

\section{Introduction}

1 L'une des missions importantes de l'université contemporaine est de répondre à la forte demande sociale d'insertion professionnelle des étudiants dans un contexte concurrentiel entre établissements d'enseignement supérieur (Musselin, 2008). Pour y parvenir, l'université se questionne sur la qualité des enseignements qu'elle dispense. Sur ce dernier point, la formation pédagogique des enseignants-chercheurs constitue un levier d'amélioration (Langevin, 2007). Malgré les efforts de soutien à l'enseignement qui se sont succédé depuis les années 1980, les enseignants-chercheurs continuent d'apprendre leur métier d'enseignant principalement «sur le tas" (Demougeot-Lebel et Perret, 2011; Knight, Tait et Yorke, 2006; Rege Colet et Berthiaume, 2009). Cette expression familière, souvent perçue avec une nuance péjorative, renvoie à un apprentissage qui s'effectue «sur le lieu de travail, par expérience directe, de manière improvisée » (Tas, s.d.). Cet article vise à dresser un panorama des pratiques d'apprentissage que l'expression «sur le tas» peut recouvrir dans le contexte de l'enseignement supérieur français et entend montrer, sur la base d'une enquête de terrain, comment les pratiques identifiées permettent aux enseignants-chercheurs novices en France de construire leurs compétences en enseignement.

2 Cet article est structuré en cinq sections. Tout d'abord, la problématique de la formation des enseignants universitaire novices est présentée. Puis, le cadre théorique composé de différents modèles d'apprentissage est explicité. Ensuite, la démarche 
méthodologique de la recherche est présentée. Puis, les résultats issus de l'enquête de terrain sont exposés. Enfin, les résultats obtenus sont discutés à la lumière d'un retour sur la littérature.

\section{La formation des universitaires novices à l'enseignement}

Dans ce travail, les enseignants universitaires novices sont des maitres de conférences ayant jusqu'à quatre ans d'ancienneté dans leur fonction. Cette définition s'inscrit dans la période du noviciat en enseignement qui peut s'étendre jusqu'à sept ans selon les contextes, les caractéristiques ou les dispositifs étudiés (Bailly, Demougeot-Lebel et Lison, 2015 ; Barrett et al., 2002 ; Demougeot-Lebel et Perret, 2010a ; Etienne et Annoot, 2014 ; King Rice, 2010 ; Ménard, 2017 ; Stes et Van Petegem, 2011).

4 La formation des enseignants-chercheurs novices à la pédagogie constitue une préoccupation d'autant plus importante pour les universités françaises qu'elle est devenue une obligation légale (Le Journal officiel de la République française [JORF], 2017). Trois principaux types de structures formelles visant la formation des nouveaux enseignants universitaires à la pédagogie se sont succédé depuis les années 1980 . D'abord, les centres d'initiation à l'enseignement supérieur étaient en charge de former les allocataires-moniteurs à l'enseignement. Depuis la loi du 8 juillet 2013 (JORF, 2013), les Écoles supérieures du professorat et de l'éducation ont, outre la responsabilité des formations initiale et continue des enseignants du primaire et du secondaire, celle de la formation pédagogique des enseignants-chercheurs. Enfin, dans le paysage de l'enseignement supérieur français, les services universitaires de pédagogie au sein des universités proposent des formations à l'enseignement et des actions de soutien aux enseignants.

Malgré la présence de structures dédiées à la formation pédagogique, le contexte français reste marqué historiquement par la faible emprise de l'institution sur les pratiques des universitaires. L'article 2 du décret $n^{\circ} 84-431$ du 6 juin 1984 (JORF, 1984) formalise la "pleine indépendance» et l'«entière liberté d'expression" des enseignants-chercheurs dans l'exercice de leur métier. En France, les universitaires revendiquent cette liberté (Potocki-Malicet, 1997), ce qui les conduit souvent à adopter des comportements très individuels (Paivandi, 2015).

6 Dans ce contexte spécifique, l'enseignant-chercheur novice s'adapte plus ou moins seul à sa mission d'enseignement, et quasiment sans formation pédagogique initiale (Altet, 2004 ; Demougeot-Lebel et Perret, 2011). Sans structure formelle ou proposition de formation, le métier d'enseignant universitaire s'apprend le plus souvent «sur le tas » (Knight et al., 2006; Rege Colet et Berthiaume, 2009). Mais que comprend cet apprentissage « sur le tas»?

7 Dans la littérature internationale, la construction des compétences des enseignants universitaires novices n'est que rarement abordée en ces termes. La construction de compétence est définie ici comme deux processus conjoints: l'apprentissage des compétences et l'acquisition des compétences. L'apprentissage décrit toute action qu'un individu peut mettre en œuvre - de manière consciente ou non - qui vise l'acquisition, c'est-à-dire les processus cognitifs d'assimilation, d'accommodation et d'équilibration (Coulet, 2011 ; Pastré, Mayen et Vergnaud, 2006 ; Piaget, 1975). 
Dans la littérature, les définitions de la compétence sont nombreuses et variées (Coulet, 2016 ; Jonnaert, 2009 ; Rey, 2014). Bien qu'il n'existe pas de définition univoque (Piot, 2008 ; Rey, 2009), il se dégage des différentes conceptualisations de la compétence des caractéristiques communes qui permettent de la définir comme l'aptitude à mobiliser de façon pertinente un ensemble de ressources appropriées en vue de traiter avec succès des situations complexes. Ainsi définie, la compétence n'est ni le savoir-faire ni la qualification. D'abord, le savoir-faire est simple alors que la compétence est complexe. Ensuite, le savoir-faire fait partie des ressources possibles d'une compétence. À ce titre, une compétence peut articuler plusieurs savoir-faire. Quant à elle, la qualification désigne les «qualités " qu'une personne doit détenir pour accomplir un travail donné (Jonnaert, 2009). La qualification se distingue de la compétence en ce qu'elle est décontextualisée et prescriptive, mais surtout par le fait qu'elle désigne les caractéristiques supposées d'un individu et non son aptitude effectivement observable à résoudre les problèmes constitutifs des situations complexes.

9 Les compétences que doivent détenir les enseignants-universitaires sont le plus souvent confinées dans des référentiels (Brassard, 2016; Centre d'appui pour l'enseignement, 2010 ; Theall et Arreola, 2006) et ne désignent pas l'approche spécifique d'une aptitude à résoudre des situations complexes. Ces référentiels présentent de manière prescriptive les ressources que doivent détenir les enseignants plutôt que des compétences (Kiffer et Tchibozo, 2013).

10 Dès lors, pour comprendre comment les enseignants universitaires novices construisent leurs compétences d'enseignement, il faut s'intéresser aux travaux traitant plus largement de leur développement professionnel. Ces travaux ont consacré beaucoup d'attention aux modalités d'apprentissage du métier (Åkerlind, 2005 ; Annoot et Fave-Bonnet, 2004 ; Demougeot-Lebel et Perret, 2010a ; Knight et al., 2006 ; Langevin, 2007 ; Ménard, 2017; Remmik, Karm, Haamer et Lepp, 2011). D’après ces travaux, l'apprentissage des universitaires novices peut s'effectuer de deux manières : soit dans le cadre de dispositifs mis en place avec l'intention de former les individus, soit via des situations ou des dispositifs dont la vocation n'est pas de les former, mais qui peuvent néanmoins exercer un effet en ce sens.

11 Dans le premier cas, un certain nombre de travaux mettent en évidence les modalités des dispositifs destinés à la formation (Langevin, 2007; Ménard, 2017; Stes et Van Petegem, 2011), les formes d'accompagnement à l'entrée dans le métier d'enseignant universitaire (Clement, Di Napoli, Annelies Gilis, Buelens, et Frenay, 2011; Ménard, 2017) et les modalités de la démarche de Scholarship of teaching and learning (Boyer, 1990 ; Rege Colet, McAlpine, Fanghanel et Weston, 2011).

12 Dans le second cas, il est souvent relevé dans la littérature que les situations ou les dispositifs informels font référence à l'apprentissage expérientiel (Kolb, 1984; Schön, 1983), l'apprentissage par expérimentation (Ballantyne, Bain et Packer, 1999) ou encore l'apprentissage « sur le tas $»^{1}$ (Knight et al., 2006).

\section{Le cadre théorique}

13 Le cadre théorique de cette recherche en sciences de l'éducation est composé de l'ensemble des modèles théoriques qui traitent de l'apprentissage du métier d'enseignant universitaire. Huit modèles ont abordé ce thème dans les travaux de 
sciences de l'éducation. Bien que de sources diverses, ces modèles présentent le point commun de chercher à comprendre comment les enseignants universitaires novices ont appris le métier d'enseignant universitaire. En ce sens, les composantes de ce cadre théorique sont homogènes. Les huit modèles identifiés sont les suivants: (1) apprentissage par observation-imitation (Bandura, 1977, 2003); (2) apprentissage collaboratif (Dillenbourg, 1999 ; Johnson et Johnson, 1983) ; (3) cadre hétéroformatif organisé (Bourgeois et Nizet, 2005); (4) tutorat (Baudrit, 2007 ; Houdé, 1996) ; (5) mentorat formel et informel (Bernatchez, Cartier, Bélisle et Bélanger, 2010); (6) consultation pédagogique (Clement et al., 2011); (7) autodidaxie (Verrier, 1999) ; (8) communautés de pratiques (Lave et Wenger, 1991 ; Wenger, 2005). Nous catégorisons ces huit modèles selon qu'ils se réfèrent à des dispositifs mis en place dans la perspective de former les individus à l'enseignement ou bien à des dispositifs - ou plus largement des situations - qui n'ont pas explicitement vocation à les former, mais qui peuvent exercer un effet en ce sens.

\subsection{Les modèles impliquant des modalités de formation et d'apprentissage fortement structurées}

La consultation désigne la démarche par laquelle les enseignants obtiennent de l'aide auprès d'un conseiller pédagogique pour leur enseignement. Cette démarche est volontaire (Morrison, 1997). Le conseiller est un spécialiste des questions relatives à l'enseignement supérieur, mais n'est pas nécessairement enseignant lui-même. La consultation pédagogique mobilise deux personnes : l'enseignant et le conseiller. La consultation se déroule au cours d'un entretien. Le conseiller peut baser ses entretiens de consultation sur les performances qui peuvent avoir été enregistrées sur divers supports (audio ou vidéo), ou peut avoir assisté directement aux enseignements. Le conseiller dispose dès lors d'un support lui permettant de commenter les performances de l'enseignant, de discuter avec lui, et, in fine, de lui prodiguer des conseils personnalisés.

Le mentorat est une relation de partenariat entre un mentor et un mentoré. Eby, Rhodes et Allen (2007) distinguent quatre dimensions constitutives du mentorat: (1) une relation entre deux individus, le mentor et le mentoré (aussi appelé protégé), dont les modes d'échanges sont idiosyncratiques; (2) un partenariat reposant sur l'apprentissage; (3) une relation définie par deux types d'aide, psychosociale et instrumentale ; (4) une relation réciproque mais asymétrique. Le mentorat peut être formel ou informel selon qu'il est défini ou non dans le cadre d'un programme de formation qui soutient et sanctionne les relations mentorales (Bernatchez et al., 2010).

Le tutorat se caractérise par une relation entre deux individus dans laquelle le tuteur apporte une aide individualisée au tutoré. La nature de l'aide diffère de celle du mentorat en ce qu'elle est exclusivement instrumentale, c'est-à-dire qu'elle repose sur les aspects techniques, matériels. Elle ne repose pas sur une relation d'affinité entre les deux partenaires et ne vise pas la création de ce type de relation. Le tutorat ne comporte pas la dimension d'aide psychosociale du mentorat (Houdé, 1996). Tout comme le mentorat formel, la dyade est formée avec l'aide d'un tiers (un programme de tutorat, une institution, une organisation, etc.). Enfin, contrairement au mentorat, la durée du tutorat est généralement limitée et déterminée par un programme (Barbier, 1996). 
17 Le cadre hétéroformatif organisé désigne les formations plus ou moins ponctuelles au cours desquelles un formateur traite des questions d'enseignement universitaire. Ces formations peuvent revêtir divers aspects comme l'utilisation de techniques d'animation, de méthodes pédagogiques, de l'utilisation d'outils spécifiques, etc. La spécificité du cadre hétéroformatif organisé dans notre cadre théorique est que le participant doit non seulement y avoir participé, mais il doit avoir vécu, au cours de la formation, un conflit socio-cognitif (Darnon, Butera et Mugny, 2008).

\subsection{Les modèles impliquant des modalités de formation et d'apprentissage faiblement structurées}

L'apprentissage par observation-imitation - aussi appelé apprentissage vicariant (Bandura 1977, 2003) repose sur l'idée que les individus ne sont pas tenus de faire systématiquement eux-mêmes les expériences perturbantes qui permettent l'apprentissage. Les individus peuvent apprendre en observant ce qu'autrui vit pour le reproduire ensuite.

19 L'autodidaxie décrit un auto-apprentissage volontaire s'effectuant en dehors de tout cadre hétéroformatif organisé où l'apprenant peut avoir ponctuellement recours à une ou plusieurs personnes-ressources (Verrier, 1999). Dans l'autodidaxie, l'autoapprentissage renvoie autant à des connaissances d'ordre savant et culturel qu'à des connaissances techniques et pratiques, mais également à des savoir-faire. Verrier (1999) précise que l'apprentissage autodidactique est volontaire pour mettre en exergue le fait qu'il s'agit d'une démarche voulue par l'apprenant. L'autodidacte est autonome dans la sélection des ressources qu'il juge nécessaires pour atteindre ses objectifs d'apprentissage. Néanmoins, il n'est pas exclu que l'apprenant "puisse demander de l'aide en cas de besoin » (Tremblay, 1986, p. 28). En effet, la définition de l'autodidaxie souligne qu'un autodidacte pourra avoir besoin de personnes-ressources en cas de « difficulté sérieuse » (Verrier, 1999, p. 89).

La théorie des communautés de pratiques défend l'idée que l'apprentissage est un acte collectif (Lave et Wenger, 1991 ; Wenger, 1998, 2005). Une communauté de pratiques est définie comme un contexte dans lequel des possibilités sont données aux membres de cette communauté de développer des compétences (Wenger, 1998). Pour qu'il y ait apprentissage au sein d'une communauté de pratiques, il faut que soient simultanément présentes trois conditions: un engagement mutuel, une entreprise commune et un répertoire partagé. Dans la conception de Wenger (1998), l'engagement mutuel correspond à l'ensemble des "relations ordinaires qui relient les membres d'une communauté en une entité sociale » (Berry, 2008, p. 29). L'entreprise commune désigne l'ensemble des actions communes. Elles sont déterminées d'une part par ce que l'institution exige en termes d'objectifs et d'activités, et d'autre part par la réalité face à laquelle ces derniers s'accomplissent, c'est-à-dire ce que les individus font concrètement dans leurs activités quotidiennes (Berry, 2008). Le répertoire partagé comprend « des routines, des mots, des outils, des procédures, des histoires, des gestes, des symboles, des styles, des actions ou des concepts créés par la communauté, adoptés au cours de son existence et devenus partie intégrante de la pratique » (Wenger, 2005, p. 91).

21 L'apprentissage collaboratif désigne une situation dans laquelle un petit groupe d'individus poursuit un but commun, ce qui permet d'optimiser les apprentissages de 
chacun (Johnson et Johnson, 1983). Autrement dit, la situation d'apprentissage par collaboration se caractérise par une relation d'interdépendance des buts d'apprentissage des individus. Dans le cas où il $\mathrm{y}$ a interdépendance, un apprenant ne peut atteindre ses buts d'apprentissage que si les autres apprenants le peuvent également. L'apprentissage coopératif s'oppose à l'apprentissage compétitif et à l'apprentissage individualiste. Dans l'apprentissage compétitif, les objectifs des apprenants sont négativement corrélés : un apprenant ne peut atteindre ses objectifs que si l'autre ne le peut pas. Dans l'apprentissage individualiste, les objectifs des apprenants sont indépendants des autres apprenants. L'apprentissage par collaboration n'est possible que si les personnes impliquées sont de même niveau cognitif, de statuts équivalents et capables de travailler ensemble à l'atteinte du but commun (Dillenbourg, 1999).

\section{La méthodologie}

L'analyse des pratiques de construction des compétences des enseignants universitaires novices de l'université en France repose sur une enquête de terrain organisée en deux phases: un questionnaire principal visant à caractériser les pratiques déclarées de construction des compétences; et des entretiens complémentaires menés auprès d'experts de la pédagogie universitaire et de l'enseignement supérieur qui visent à mettre en perspective les résultats du questionnaire.

Le questionnaire principal (présenté en annexe) comporte 50 questions réparties en quatre catégories. La première catégorie de questions vise à identifier les caractéristiques pertinentes du répondant (âge, sexe, discipline(s) de rattachement, ancienneté). Ensuite, des questions sont formulées dans le but de relever si les différents modèles composant le cadre théorique sont effectivement utilisés, selon quelles modalités précises, à quelle fréquence et par quel profil d'enseignantchercheur. La troisième série de questions propose aux répondants d'ordonner les modèles utilisés par leur importance perçue dans la pratique. Enfin, une série de questions porte sur l'auto-évaluation des compétences. Pour pouvoir être évaluée, la compétence est décomposée ici en cinq aptitudes, entendues ici au sens de capacités élémentaires. Le terme aptitude est délibérément employé pour le distinguer de savoirfaire ou de compétences élémentaires qui désignent des ressources de la compétence. Les cinq aptitudes s'organisent de la manière suivante:(1) identifier les problèmes à résoudre dans les situations complexes; (2) identifier les ressources pertinentes à mettre en œuvre; (3) sélectionner et combiner ces ressources; (4) utiliser les ressources dans la situation concrète; (5) s'assurer du succès dans la maîtrise de la situation. Ces aptitudes s'articulent entre elles de façon successive et conditionnelle. Autrement dit, une aptitude ne s'active que lorsque la précédente l'a été. Cette décomposition séquentielle de la compétence est de nature analytique et opérationnelle. Elle n'a ni vocation à réduire la complexité que recouvre le concept de compétence, ni la singularité des situations auxquelles se confrontent les individus. La méthode utilisée pour déterminer s'il y a eu construction des compétences consiste à mesurer l'écart entre les scores d'auto-efficacité au moment du recrutement et ceux au moment de la passation du questionnaire pour chacune de ces aptitudes. Les scores d'auto-efficacité sont des notes attribuées par les répondants sur une échelle de 0 à 100 en fonction de leur sentiment de maîtriser telle ou telle aptitude. Le choix de cette 
méthode repose sur l'affirmation de Bandura (2006) selon laquelle plus le sentiment d'efficacité personnelle à réaliser une activité est fort, plus la probabilité que l'individu accomplisse cette activité avec succès est élevée.

La passation du questionnaire s'est faite via une plateforme dédiée aux enquêtes par questionnaire sur Internet et s'est tenue de novembre 2015 à février 2016. Sa diffusion a permis de collecter les réponses d'un échantillon de 376 maitres de conférences issus d'une population-mère de 5395 maîtres de conférences novices, recrutés de 2011 à 2014. Après redressement par pondération, l'échantillon final composé de 376 individus a été rendu représentatif de la répartition de la population-mère selon le genre et les grandes disciplines.

Puis, des entretiens complémentaires ont été menés auprès de quatre experts de l'enseignement supérieur de l'enseignement supérieur et de la recherche (tableau 1). Ces derniers ont accepté d'évaluer les résultats du questionnaire. Leurs positions au sein de différentes institutions universitaires françaises ont permis de produire une analyse éclairée par des visions complémentaires. Cette évaluation des pratiques a également permis d'alimenter la discussion des résultats obtenus à l'issue de l'enquête par questionnaire.

Tableau 1. Champs d'expertise des experts interrogés

\begin{tabular}{|c|c|}
\hline EXPERTS & FONCTIONS \\
\hline 1 & Responsable d'un centre de pédagogie universitaire \\
\hline 2 & Responsable d'un centre de pédagogie universitaire \\
\hline 3 & $\begin{array}{c}\text { Chargé de mission pour la commission de la formation et de l'insertion professionnelle } \\
\text { auprès de la Conférence des Présidents d'Université (CPU) }\end{array}$ \\
\hline 4 & $\begin{array}{c}\text { Experte auprès de la Mission de la Pédagogie et du Numérique pour l'Enseignement } \\
\text { Supérieur (MiPNES) }\end{array}$ \\
\hline
\end{tabular}

\section{Les résultats de l'enquête par questionnaire}

L'enquête de terrain a permis d'obtenir cinq catégories de résultats : (1) les fréquences d'utilisation des différents modèles; (2) leurs modalités d'utilisation; (3) leur répartition dans l'échantillon selon le genre et la grande discipline (Droit, Lettres, Sciences et Médecine) ; (4) leurs combinaisons ; (5) les scores d'auto-efficacité des cinq aptitudes constitutives de la compétence.

\subsection{Les fréquences d'utilisation des modèles}

Les résultats montrent que les modèles les plus utilisés sont l'autodidaxie, l'apprentissage par observation-imitation et l'apprentissage par collaboration et que les modèles les moins utilisés sont la consultation pédagogique et le tutorat. Cette hiérarchie dans la fréquence d'utilisation se vérifie dans les trois méthodes de classement utilisées (tableau 2 ci-dessous). Le premier classement repose sur le 
traitement individuel des réponses à l'utilisation des modèles. Par exemple, si un novice a déclaré avoir recouru à l'autodidaxie, il est comptabilisé dans le calcul de la fréquence totale de ce modèle. Le deuxième classement repose sur le traitement des réponses à une question proposant aux répondants de classer les modèles utilisés par ordre d'importance. Seuls les modèles classés en première position sont retenus. Enfin, le troisième classement repose sur les premières et deuxièmes places cumulées.

Tableau 2. Trois classements des modèles par fréquence d'utilisation

\begin{tabular}{|c|c|c|c|}
\hline \multirow{2}{*}{ Modèles } & \multicolumn{3}{|c|}{ Rang } \\
\hline & $\begin{array}{l}\text { Traitement } \\
\text { individuel }\end{array}$ & $\begin{array}{c}1^{\mathrm{er}} \text { selon les } \\
\text { novices }\end{array}$ & $\begin{array}{c}1^{\mathrm{er}}+2^{\text {ème }} \text { selon les } \\
\text { novices }\end{array}$ \\
\hline Apprentissage par observation-imitation & 1 & 1 & 1 \\
\hline Autodidaxie & 2 & 2 & 2 \\
\hline Apprentissage par collaboration & 3 & 3 & 3 \\
\hline Communauté de pratiques & 5 & 5 & 4 \\
\hline Mentorat & 4 & 4 & 5 \\
\hline $\begin{array}{l}\text { Cadre hétéroformatif avec interactions et } \\
\text { conflits socio-cognitifs }\end{array}$ & 7 & 8 & 6 \\
\hline Consultation pédagogique & 6 & 6 & 7 \\
\hline Tutorat & 8 & 7 & 8 \\
\hline
\end{tabular}

Les modèles de tête mettent en évidence la tendance des novices à recourir plus fréquemment à des situations ou des dispositifs dont la vocation n'est pas de les former, mais qui peuvent exercer un effet en ce sens. En outre, les novices ne semblent pas privilégier des dispositifs mis en place avec l'intention de les former aux activités d'enseignement (consultation pédagogique, tutorat).

Ces résultats trouvent écho dans la littérature qui montre que l'apprentissage du métier d'enseignant du supérieur se réalise de manière informelle (Knight et al., 2006). Pour autant, nos résultats viennent questionner une partie de la littérature selon laquelle l'apprentissage du métier s'effectue relativement seul (Altet, 2004). En fait, il n'y a que deux modèles où l'apprenant est relativement seul. Nos résultats permettent de constater que le recours à autrui n'est pas une pratique marginale chez les novices, l'apprentissage par collaboration arrive en troisième position des classements des modèles les plus utilisés; l'autodidaxie, qui arrive en deuxième position des classements, n'exclut pas le recours à des personnes-ressources (tableau 2). 


\subsection{Les modalités d'utilisation des modèles}

30 L'utilisation d'un modèle ou d'un autre ne semble pas reposer sur le hasard. Au contraire, il semblerait qu'une logique se dégage du choix des modèles selon les objets sur lesquels ils ont porté. Les résultats montrent que chaque modèle d'apprentissage peut répondre à des besoins d'apprentissage spécifiques, même si certaines modalités sont représentées dans plusieurs modèles. Les enseignants-chercheurs novices combinent les modèles en fonction des besoins d'apprentissage éprouvés. Le tableau 3 ci-dessous récapitule les objets principaux relatifs à l'utilisation de chaque modèle, selon les répondants.

Tableau 3. Modalités d'utilisation des modèles

\begin{tabular}{|c|c|}
\hline Modèles & Objets sur lesquels porte le modèle - Modalités \\
\hline $\begin{array}{l}\text { Apprentissage par } \\
\text { observation-imitation }\end{array}$ & $\begin{array}{l}\text { - Tout type d'activité d'enseignement } \\
\text { - Techniques de présentation orale empruntées à d'autres domaines que } \\
\text { l'enseignement }\end{array}$ \\
\hline $\begin{array}{l}\text { Apprentissage par } \\
\text { collaboration }\end{array}$ & $\begin{array}{l}\text { - Co-animation d'enseignement } \\
\text { - Co-organisation } \\
\text { - Projet pédagogique commun }\end{array}$ \\
\hline $\begin{array}{l}\text { Formation (cadre } \\
\text { hétéroformatif organisé) }\end{array}$ & $\begin{array}{l}\text { - Techniques de présentation orale : utilisation de la voix, improvisation, } \\
\text { parler en public, etc. } \\
\text { - Méthodes pour accroître l'interactivité des enseignements (pédagogies } \\
\text { actives, apprentissage par résolution de problèmes, enseignement à un } \\
\text { grand groupe, etc.) } \\
\text { - Maîtrise des outils pour l'enseignement (TICE) et pour l'évaluation } \\
\text { - Connaissance de l'étudiant (ses caractéristiques, ses attentes, la gestion } \\
\text { des conflits, théories de l'apprentissage) }\end{array}$ \\
\hline Tutorat & $\begin{array}{l}\text { - Modalités d'évaluation } \\
\text { - Mise en place des examens }\end{array}$ \\
\hline Mentorat formel & - Stratégie d'enseignement et d'évaluation \\
\hline Mentorat informel & $\begin{array}{l}\text { - Gestion des problèmes avec les étudiants } \\
\text { - Utilisation de supports pédagogiques (documents, ouvrages, etc.) }\end{array}$ \\
\hline $\begin{array}{l}\text { Communauté de } \\
\text { pratiques }\end{array}$ & $\begin{array}{l}\text { - Gestion et administration des formations universitaires } \\
\text { - Innovation dans l'enseignement } \\
\text { - Utilisation des TICE } \\
\text { - Sorties pédagogiques (stages, voyages, etc.) } \\
\text { - Recherche (enseignement de la méthodologie de recherche, diffusion et } \\
\text { valorisation de la recherche) }\end{array}$ \\
\hline
\end{tabular}




\begin{tabular}{|l|l|}
\hline Autodidaxie & $\begin{array}{l}\text { - Identification d'un problème dans une situation } \\
\text { - Identification de ressources potentielles pour le résoudre }\end{array}$ \\
\hline $\begin{array}{l}\text { Consultation } \\
\text { pédagogique }\end{array}$ & $\begin{array}{l}\text { - Dispositifs d'évaluation } \\
\text { - Gestion des problèmes avec les étudiants (conflits, etc.) } \\
\text { - Utilisation des TICE } \\
\text { - Innovation dans l'enseignement }\end{array}$ \\
\hline
\end{tabular}

\subsection{La répartition des modèles dans l'échantillon}

31 Parmi les caractéristiques personnelles des répondants, les variables «sexe » et "grande discipline» permettent de décomposer l'échantillon en huit profils homogènes. A partir de ces profils, des comparaisons peuvent être établies. La répartition des modèles par profil (tableau 4 ci-dessous) permet d'observer que l'apprentissage par observation-imitation et l'autodidaxie font partie des comportements dominants (sept profils sur huit). En revanche, la consultation pédagogique n'est présente que chez les femmes en médecine, pharmacie et odontologie en deuxième position du classement. Notons également que le tutorat et le cadre hétéroformatif sont tous deux absents des comportements dominants des huit profils.

Lorsque l'on observe les comportements par discipline, l'ordre de préférence des modèles varie. En lettres et sciences humaines, l'autodidaxie arrive en première position alors qu'en sciences juridiques, politiques, économiques et de gestion et en médecine, pharmacie et odontologie, l'apprentissage par observation-imitation se classe premier. En sciences et techniques, l'autodidaxie est le modèle le plus utilisé par les femmes alors que l'apprentissage par observation-imitation est le modèle le plus plébiscité par les hommes.

Tableau 4. Comportement d'apprentissage et profil d'enseignant-chercheur novice

\begin{tabular}{|c|c|c|c|c|}
\hline Profils types & $\begin{array}{c}\text { Modèles classés } \\
\text { en } \mathbf{1}^{\text {ère }} \text { position }\end{array}$ & $\begin{array}{c}\text { Modèles classés } \\
\text { en } \mathbf{2}^{\text {ème }} \text { position }\end{array}$ & $\begin{array}{c}\text { Modèles classés } \\
\text { en } \mathbf{3}^{\text {ème }} \text { position }\end{array}$ & $\begin{array}{c}\text { Modèles classés } \\
\text { en } \mathbf{4}^{\text {ème }} \text { position }\end{array}$ \\
\hline $\begin{array}{c}\text { Femme en lettres et } \\
\text { sciences humaines }\end{array}$ & autodidaxie & $\begin{array}{c}\text { observation- } \\
\text { imitation }\end{array}$ & collaboration & $\begin{array}{c}\text { communautés de } \\
\text { pratiques }\end{array}$ \\
\hline $\begin{array}{c}\text { Homme en lettres et } \\
\text { sciences humaines }\end{array}$ & autodidaxie & $\begin{array}{c}\text { observation- } \\
\text { imitation }\end{array}$ & collaboration & \\
\hline $\begin{array}{c}\text { Femme en sciences et } \\
\text { techniques }\end{array}$ & autodidaxie & $\begin{array}{c}\text { observation- } \\
\text { imitation }\end{array}$ & collaboration & \\
\hline $\begin{array}{c}\text { Homme en sciences et } \\
\text { techniques }\end{array}$ & observation- \\
imitation & autodidaxie & & \\
\hline
\end{tabular}




\begin{tabular}{|c|c|c|l|l|}
\hline $\begin{array}{c}\text { Femme en médecine, } \\
\text { pharmacie et } \\
\text { odontologie }\end{array}$ & $\begin{array}{c}\text { observation- } \\
\text { imitation }\end{array}$ & $\begin{array}{c}\text { - mentorat } \\
\text { - autodidaxie } \\
\text { - consultation } \\
\text { pédagogique }\end{array}$ & & \\
\hline $\begin{array}{c}\text { Homme en médecine, } \\
\text { pharmacie et } \\
\text { odontologie }\end{array}$ & $\begin{array}{c}\text { observation- } \\
\text { imitation }\end{array}$ & - autodidaxie & & \\
\hline $\begin{array}{c}\text { Femme en sciences } \\
\text { juridiques, politiques, } \\
\text { économiques et de } \\
\text { gestion }\end{array}$ & $\begin{array}{c}\text { observation- } \\
\text { imitation }\end{array}$ & - mentorat & & \\
\hline $\begin{array}{c}\text { Homme en sciences } \\
\text { juridiques, politiques, } \\
\text { économiques et de } \\
\text { gestion }\end{array}$ & $\begin{array}{c}\text { observation- } \\
\text { imitation }\end{array}$ & collaboration & autodidaxie & \\
\hline
\end{tabular}

Les comportements d'apprentissage des enseignants-chercheurs novices permettent à nouveau de conclure que les modèles d'apprentissage faiblement structurés sont les plus fortement sollicités (l'autodidaxie et l'apprentissage par observation-imitation), alors que les modèles fortement structurés sont rarement utilisés (consultation pédagogique), voire totalement absents des comportements dominants (tutorat et cadre hétéroformatif organisé).

\subsection{Des combinaisons de modèles}

L'analyse des combinaisons de modèles montre que l'autodidaxie est associée avec d'autres modèles dans $41,03 \%$ des utilisations, ce qui en fait le modèle le plus souvent présent dans les associations. Il ressort également que la paire collaborationautodidaxie est présente dans les deux combinaisons les plus utilisées, soit dans 26,12\% des utilisations combinées. Le triplet imitation-collaboration-autodidaxie représente près de $18 \%$ des utilisations. Enfin, le tutorat et la consultation pédagogique n'apparaissent pas dans les dix premières combinaisons les plus fréquentes.

$\mathrm{Au}$ vu de la place qu'occupe l'autodidaxie dans les combinaisons, les résultats semblent indiquer que les enseignants novices privilégient l'autonomie dans leur pratique. Il n'est pas exclu que ce choix lui-même soit contraint par un contexte de solitude. Bien que l'autodidaxie permette le recours ponctuel à une ou plusieurs personne-ressources, ce modèle faiblement structuré présente des limites qui peuvent conduire les novices à recourir à d'autres modèles, en particulier à l'apprentissage par collaboration et à l'apprentissage par observation-imitation.

\subsection{La construction des compétences et les scores d'auto-efficacité}

Le calcul de la différence des scores d'auto-efficacité a été appliqué de manière systématique aux cinq aptitudes constitutives de la construction des compétences. On observe une progression du sentiment d'auto-efficacité pour toutes les activités de tous 
les modèles. En revanche, aucune de ces progressions n'est statistiquement significative, et ce, quel que soit le modèle utilisé individuellement. En revanche, ce calcul a été effectué pour les trois combinaisons de modèles les plus utilisées (tableau 5 ci-dessous). Il en ressort que la combinaison 3 «observation/imitation - communauté de pratiques - autodidaxie » est significativement estimée plus efficace que les deux autres.

Tableau 5. Combinaisons de modèles

\begin{tabular}{|c|c|c|}
\hline $\begin{array}{l}\text { Combinaison } 1 \\
\text { Observation/imitation } \\
\text { collaboration - autodidaxie }\end{array}$ & $\begin{array}{l}\text { Combinaison } 2 \\
\text { Communauté de pratiques - } \\
\text { collaboration - autodidaxie }\end{array}$ & $\begin{array}{l}\text { Combinaison } 3 \\
\text { Observation/imitation } \\
\text { communauté de pratiques } \\
\text { autodidaxie }\end{array}$ \\
\hline
\end{tabular}

\section{La discussion des résultats}

Les résultats de l'enquête par questionnaire ont permis de mettre en évidence : (1) la prédominance des comportements autonomes chez les novices; (2) l'efficacité des modèles d'apprentissage et de leur combinaison; (3) les comportements d'apprentissage selon les profils d'enseignants-chercheurs novices.

\subsection{La prédominance des comportements autonomes}

L'enquête a révélé que les modèles aux modalités faiblement structurées sont les plus utilisés par les novices. Ces derniers déclarent apprendre le métier d'enseignant du supérieur au travers de situations ou de dispositifs dont la vocation principale n'est pas de les former, mais qui peuvent exercer un effet en ce sens. Parmi eux, l'autodidaxie auto-apprentissage volontaire s'effectuant en dehors de toute cadre hétéroformatif organisé, dans lequel le recours à une personne-ressource est possible (Verrier, 1999) est le plus plébiscité. Deux explications nous semblent plausibles et de nature à être discutées : la rémanence de la culture universitaire et l'appartenance générationnelle des novices.

La culture universitaire semble jouer un rôle important dans la perpétuation du comportement autonome et principalement informel des novices dans leur processus d'acquisition de compétences d'enseignement. A ce propos, des chercheurs nordaméricains (Bowen et Schuster, 1986 ; Clark, 1987 ; Finkelstein, 1982) ont mis au jour les dimensions spécifiques de la culture universitaire. Bowen et Schuster (1986) notent que trois valeurs caractérisent le métier d'universitaire : la poursuite libre du savoir en tant que vérité ; le besoin perpétuel de liberté de pensée ; la volonté agissante de participer de façon collégiale à la vie universitaire. Pour Clark (1987), le métier d'universitaire se définit par trois caractéristiques communes : l'individualisme, la liberté professionnelle et la concurrence. Finkelstein (1982) identifie des traits psychologiques communs à tous les universitaires en contexte nord-américain: le goût du développement intellectuel, le désir d'autonomie professionnelle, le besoin d'accomplissement personnel, le désir de servir la communauté et l'attachement à certains principes d'excellence, de compétition et de mérite. Ces trois recherches, bien que datant, 
permettent de cerner les valeurs de la culture universitaire contemporaine : liberté d'agir, de s'organiser, de penser, etc. Les pratiques d'apprentissage du métier de chercheur sont éminemment inscrites dans ce contexte. Il est raisonnable de concevoir que l'apprentissage du métier de chercheur, inscrit dans ce système de valeurs prônant l'autonomie et la liberté, façonneraient également le comportement d'apprentissage des universitaires novices à leur métier d'enseignant du supérieur. Cependant, ces recherches menées dans le contexte nord-américain ne représentent pas nécessairement les "particularités» de la situation française. La présente recherche n'a pas traité cette question qui constitue une piste de recherche ultérieure. Cette piste pourra prendre en considération les particularités françaises dont on peut supposer qu'elles exercent une influence sur la tendance à l'autonomie, telles que la double hiérarchie: statut des universitaires (professeurs des universités et maîtres de conférences), statut des enseignements (TP-TD-CM) ou encore la survalorisation de la recherche en début de carrière.

40 L'hypothèse selon laquelle les pratiques d'apprentissage sont spécifiques à la génération $\mathrm{Y}$ pourrait a priori permettre d'expliquer les comportements tendanciellement autonomes des novices. La génération Y comprend les individus nés entre 1979 et 1995 (Demougeot-Lebel, 2014). Diverses recherches (Eisner, 2005 ; Josiam et al., 2009) ont permis de dégager des caractéristiques psychosociologiques associées à cette classe d'âge, en particulier la recherche de sens au travail et le besoin d'accomplissement, le travail collaboratif et l'esprit d'équipe, la liberté d'expression, les compétences élaborées dans le domaine des technologies de l'information et de la communication. Une étude menée sur les pratiques d'enseignement des enseignantschercheurs de la génération $Y$ montre que l'effet de génération attendu est absent des pratiques (Demougeot-Lebel, 2014). Du reste, les générations précédentes elles-mêmes avaient appris le métier d'enseignant "sur le tas ", faisant ainsi preuve d'une certaine autonomie. Peut-être s'agissait-il d'une autonomie d'une autre nature, plus contrainte par l'absence alors plus marquée de structures d'encadrement. Quoi qu'il en soit, cette recherche reste à faire car la littérature sur les pratiques d'apprentissage des enseignants-chercheurs de la génération Y est à notre connaissance muette.

\subsubsection{L'efficacité des modèles et des combinaisons de modèles}

41 Nos résultats indiquent que tous les modèles apparaissent également efficaces pour construire les compétences d'enseignement, à l'exclusion de l'autodidaxie et de l'apprentissage par collaboration dont l'efficacité apparaît plus limitée. À notre connaissance, la littérature scientifique ne traite pas de l'efficacité des pratiques de construction des compétences des universitaires en ces termes précis. Cette dernière traite majoritairement des relations entre conceptions de l'enseignement et de l'apprentissage d'une part, et pratiques d'enseignement et d'apprentissage d'autre part. Dans ce domaine, les recherches montrent l'efficacité des modèles fortement structurés sur les conceptions et pratiques d'enseignement des novices (Bailly et al., 2015; Demougeot-Lebel et Perret, 2010b ; Ménard, 2017).

D'une manière générale, il semblerait que les apprentissages peu guidés (correspondant dans notre conceptualisation aux modèles faiblement structurés) sont significativement moins efficaces que les apprentissages guidés (correspondant chez nous aux modèles fortement structurés) (Kirschner, Sweller et Clark, 2006). Ces recherches montrent cependant que les apprenants possédant un important bagage de 
connaissances bénéficient autant d'une forte guidance que d'une faible guidance (Kirschner et al., 2006, p. 83-84). Les universitaires se trouvent précisément dans ce cas, leur niveau d'études, leur degré d'autonomie, leurs aptitudes métacognitives et leur pratique réflexive en font des apprenants déjà efficaces. Pourtant, ce sont les modèles faiblement guidés qui sont les moins bien évalués par les novices. Les facteurs de cette inefficacité restent à déterminer. Plusieurs pistes sont à explorer, notamment celle de l'éloignement thématique des enseignements confiés aux novices. En d'autres termes, le sentiment d'efficacité peut-il décroître à mesure que la thématique de l'enseignement en charge diverge de la spécialité du novice?

\subsection{Les profils de novices et comportement d'apprentissage}

L'apprentissage par observation-imitation est un comportement présent chez tous les novices, quel que soit leur profil. Ce résultat rejoint ceux d'Étienne et Annoot (2014) qui, dans une recherche sur l'entrée dans le métier d'universitaire en France, ont également noté la tendance des enseignants-chercheurs à imiter les pratiques d'enseignement de leurs pairs. Outre les modèles compris initialement dans le cadre théorique de notre recherche, les discussions informelles avec les collègues sont apparues comme une modalité d'apprentissage importante. Ces discussions prennent principalement place dans des lieux et lors de moments informels (pauses "café », au hasard de rencontres dans les couloirs de l'université, lors des déjeuners, etc.). Selon Bandura (1977), les apprentissages par observation-imitation peuvent être optimisés quand ils sont précédés de renforcements, c'est-à-dire par le fait qu'un apprenant connaisse les résultats d'un comportement. Le renforcement peut prendre la forme d'un modelage verbal: prendre connaissance, par un récit de pratiques, de leur efficacité. Donc, écouter ou lire le récit de pratiques d'un collègue au cours de discussions ou d'échanges informels peut permettre d'optimiser les apprentissages par observation-imitation. Le modelage verbal est d'autant plus efficace que les capacités conceptuelles et verbales des apprenants sont développées (Bandura, 1977), ce qui est précisément le cas des enseignants-chercheurs.

Les différences de comportement d'apprentissage selon les profils peuvent s'expliquer par les spécificités épistémologiques et traditionnelles des disciplines auxquelles sont rattachés les universitaires. Berthiaume (2007) met en évidence l'existence d'un savoir pédagogique disciplinaire propre à chaque discipline. Par ailleurs, Endrizzi (2011) relève que l'expression des besoins de formation peut varier selon la discipline d'appartenance. Par exemple, en sciences et techniques et en médecine, les demandes de formation semblent, d'après l'auteure, porter sur l'utilisation des technologies de l'information et de la communication, alors qu'en lettres et sciences humaines, elles portent davantage sur des questions de relations avec les étudiants.

\section{Conclusion}

Les résultats de cette recherche sur la construction de compétences des universitaires novices entendent contribuer à une meilleure connaissance des processus d'apprentissage du métier d'enseignant à l'université. L'expression "sur le tas " désignant familièrement un apprentissage «sur le lieu de travail, par expérience directe, de manière improvisée " est souvent convoquée dans la littérature pour 
répondre à cette question. Ce travail a déconstruit l'expression «sur le tas " pour faire apparaître les différentes pratiques d'apprentissage que les enseignants universitaires déploient pour construire leurs compétences d'enseignement. Il apparaît que, bien que les pratiques de construction des compétences des novices soient variées, non structurées et principalement informelles, elles n'en relèvent pas moins de modèles précis, bien identifiés et distincts.

Le décret 2017-854 du 9 mai 2017 (JORF, 2017) prévoit que les maîtres de conférences bénéficient «d'une formation visant l'approfondissement des compétences pédagogiques nécessaires à l'exercice du métier » (article 13) au cours de la première année suivant leur prise de fonction. Cette formation obligatoire d'un volume équivalent à un sixième de leur service d'enseignement annuel (soit 32 heures) " peut tenir compte de leur parcours antérieur et être accompagnée d'un tutorat » (article 13). Le décret n'impose ni les modalités de formation, ni les compétences visées par la formation. La formulation flexible du texte permet aux universités de l'appliquer selon les modalités qu'elles jugeront adaptée. Dans ce contexte, nos résultats, montrant la prévalence des comportements autonomes chez les novices, peuvent contribuer à la réflexion des universités en matière de formation à l'enseignement. La recherche doit se poursuivre pour déterminer les raisons précises de ces comportements autonomes, raisons à prendre en compte aussi dans la réflexion. En attendant, il faut mentionner l'intérêt des pouvoirs publics pour ce sujet, qui constitue un certain encouragement pour la recherche en pédagogie universitaire en France.

\section{BIBLIOGRAPHIE}

Åkerlind, G. (2005). Academic growth and development - How do university academics experience it? Higher Education, 50, 1-32.

Altet, M. (2004). Enseigner en premier cycle universitaire : Des formes émergentes d'adaptation ou de la « métis » enseignante. Dans E. Annoot et M.-F. Fave-Bonnet (éd.), Pratiques pédagogiques dans l'enseignement supérieur : enseigner, apprendre, évaluer. Paris : L'Harmattan.

Annoot, E. et Fave-Bonnet, M.-F. (2004). Pratiques pédagogiques dans l'enseignement supérieur : enseigner, apprendre, évaluer. Paris : L'Harmattan.

Bailly, B., Demougeot-Lebel, J. et Lison, C. (2015). La formation d'enseignants universitaires nouvellement recrutés : quelles retombées ? Revue internationale de pédagogie de l'enseignement supérieur, 31(3). http://journals.openedition.org/ripes/990.

Ballantyne, R., Bain, J. D. et Packer, J. (1999). Researching university teaching in Australia: Themes and issues in academics' reflections. Studies in Higher Education, 2(24), 237-257.

Bandura, A. (1977). Social learning theory. Englewood Cliffs, NJ: Prentice-Hall.

Bandura, A. (2003). Auto-efficacité. Le sentiment d'efficacité personnelle. Paris : De Boeck Université.

Bandura, A. (2006). Guide for constructing self-efficacy scales. Dans F. Pajares et T. Urdan (éd.), Self-efficacy beliefs of adolescents (Vol. 5, p. 307-337). Greenwich, CT : Information Age Publishing. 
Barbier, J.-M. (1996). Tutorat et fonction tutorale : quelques entrées d'analyse. Recherche et Formation, 22, 7-19.

Barrett, J., Jones, G., Mooney, E., Thornton, C., Cady, J., Guinee, P. et Olson, J. (2002). Working with novice teachers: challenges for professional development. Mathematics Teacher Education and Development, 4, 15-27.

Baudrit, A. (2007). Le tutorat. Richesses d'une méthode pédagogique. Louvain-la-Neuve : De Boeck Supérieur.

Bernatchez, P.-A., Cartier, S., Bélisle, M. et Bélanger, C. (2010). Le mentorat en début de carrière : retombées sur la charge professorale et condition de mise en œuvre d'un programme en milieu universitaire. Revue internationale de pédagogie de l'enseignement supérieur, 26(1). http:// ripes.revues.org/374.

Berry, V. (2008). Les communautés de pratiques : note de synthèse. Pratiques de formation: Analyses, $54,12-47$.

Berthiaume, D. (2007). A description of discipline-specific pedagogical knowledge (DPK) encountered in the discourse of four university professors from four different disciplinary areas. (Thèse de doctorat) Université McGill, Montréal.

Bourgeois, E. et Nizet, J. (2005). Apprentissage et formation des adultes. Paris : Presses universitaires de France.

Bowen, H. et Schuster, J. (1986). American Professors. A National Resource Imperiled. Oxford: University Press.

Boyer, E. (1990). Scholarship Reconsidered: Priorities for the Professoriate. Princeton, NJ: The Carnegie Foundation for the Advancement of Teaching.

Brassard, N. (2016). Profil de compétences de l'enseignant de niveau universitaire. Montréal : Ecole nationale de l'administration publique (ENAP).

Centre d'Appui Pour l'Enseignement (2010). Compétences pour enseigner. Repéré à http:// imedia.emn.fr/wp/?page_id=585. Consulté le 16 juin 2014.

Clark, B.R. (1987). The academic life. Small worlds, different worlds. Princeton, NJ: Carnegie Foundation for the Advancement of Teaching.

Clement, M., Di Napoli, R., Annelies Gilis, A., Buelens, H. et Frenay, M. (2011). Educational consultation for reflective-dialogic partnerships : a possible model. Recherche et Formation, 67, 31-47.

Coulet, J.-C. (2011). La notion de compétence : un modèle pour décrire, évaluer et développer les compétences. Le travail humain, 74(1), 1-30.

Coulet, J.-C. (2016). Les notions de compétence et de compétences clés : l'éclairage d'un modèle théorique fondé sur l'analyse de l'activité. Activités, 13(1). http://activites.revues.org/2745.

Darnon, C., Butera, F. et Mugny, G. (2008). Des conflits pour apprendre. Grenoble : Presses Universitaires de Grenoble.

Demougeot-Lebel, J. et Perret, C. (2010a). Identifier les conceptions de l'enseignement et de l'apprentissage pour accompagner le développement professionnel des enseignants débutant à l'université. Savoir, 23, 55-65. 
Demougeot-Lebel, J. et Perret, C. (2010b). Les chargés de TD/TP ont-ils tous les mêmes inquiétudes avant leurs premières interventions face aux étudiants ? Recherches en éducation, 9 , 99-113.

Demougeot-Lebel, J. et Perret, C. (2011). Qu'attendent les enseignants universitaires français en termes de formation et d'accompagnement pédagogiques? Revue internationale de pédagogie de l'enseignement supérieur, 27(1). http://ripes.revues.org/456.

Demougeot-Lebel, J. (2014). Enseignants-chercheurs de la Génération Y : incidence sur les pratiques pédagogiques ? Revue internationale de pédagogie de l'enseignement supérieur, 3(30). http:// ripes.revues.org/883.

Dillenbourg, P. (1999). What do you mean by « collaborative learning »? Dans P. Dillenbourg (éd.), Collaborative learning: Cognitive and computational approaches (p. 1-19). Oxford: Pergamon.

Eby, L., Rhodes, J. et Allen, T. (2007). Evolution and definition of mentoring. Dans T. Allen et L. Eby (éd.), The Blackwell Handbook of Mentoring. A multiple Perspectives Approach. Malden: Blackwell Publishing Ltd.

Eisner, S.P. (2005). Managing Generation Y. SAM Advanced Management Journal, 70(4), 4-15.

Endrizzi, L. (2011). « Savoir enseigner dans le supérieur : un enjeu d'excellence pédagogique ». Dossier d'actualité Veille et analyses, (64), septembre. Repéré à http://www.inrp.fr/vst/DA/ detailsDossier.php?dossier=64etlang=fr.

Étienne, R. et Annoot, E. (2014). L'entrée dans le métier d'enseignant-chercheur. Article présenté au $28^{\mathrm{e}}$ Congrès de l'AIPU, Mons.

Finkelstein, M. (1982). The American Academic Profession. A Synthesis of Social Scientific Inquiry since World War II. Columbus, OH : Ohio State University Press.

Houdé, R. (1996). Le mentor : transmettre un savoir-être. Revigny-sur-Ornain : Hommes et Perspectives, Théorie et Pratique.

Johnson, R. T. et Johnson, D. W. (1983). Effects of cooperative, competitive, and individualistic learning experiences on social development. Exceptional Children, 49(4), 323-329.

Jonnaert, P. (2009). Compétences et socioconstructivime. Un cadre théorique. Bruxelles : De Boeck. Josiam, B.M., Crutsinger, C., Reynolds, J.S., Dotter, T., Thozhur, S., Baum, T. et Devine, F. G. (2009). An Empirical Study of the Work Attitudes of Generation Y College Students in the USA: The Case of Hospitality and Merchandising Undergraduate Majors. Journal of Services Research, 9(1), 5-30.

King Rice, J. (2010). The Impact of Teacher Experience: Examining the Evidence and Policy Implications. Rapport de l'Urban Institute, National Center for Analysis of Longitudinal Data in Education Research (CALDER). ERIC: ED565612.

Kiffer, S. et Tchibozo, G. (2013). Developing the teaching competences of a novice faculty member: a review of international literature. Policy Futures in Education, 11(3), 277-289.

Kirschner, P., Sweller, J. et Clark, R. (2006). Why minimal guidance during instruction does not work: an analysis of the failure of constructivist, discovery, problem-based, experiential, and inquiry-based teaching. Educational Psychologist, 41(2), 75-86

Knight, P., Tait, J. et Yorke, M. (2006). The professional learning of teachers in higher education. Studies in Higher Education, 31(3), 319-339.

Kolb, D. A. (1984). Experiential learning: Experience as the source of learning and development. Englewood Cliffs, NJ : Prentice-Hall. 
Langevin, L. (2007). Formation et soutien à l'enseignement universitaire. Des constats et des exemples pour inspirer l'action. Québec: Presses de l'université du Québec.

Lave, J. et Wenger, E. (1991). Situated Learning: Legitimate Peripheral Participation. Cambridge : Cambridge University Press.

Le Journal officiel de la République française [JORF] (1984). Décret $n^{\circ} 84-431$ du 6 juin 1984 fixant les dispositions statutaires communes applicables aux enseignants-chercheurs et portant statut particulier $d u$ corps des professeurs des universités et du corps des maitres de conférences. Version consolidée au 18 novembre 2018. Repéré à https://www.legifrance.gouv.fr/affichTexte.do ?

cidTexte =JORFTEXT000000520453\#LEGIARTI000020558078.

Le Journal officiel de la République française [JORF] (2013). LOI n 2013-595 du 8 juillet 2013 d'orientation et de programmation pour la refondation de l'école de la République (1). Repéré à https:// www.legifrance.gouv.fr/affichTexte.do?

cidTexte =JORFTEXT000027677984etfastPos =1etfastReqId =862354592etcategorieLien =cidetoldAction =rechTexte

Le Journal officiel de la République française [JORF] (2017). Décret no 2017-854 du 9 mai 2017 modifiant le décret no 84-431 du 6 juin 1984 modifié fixant les dispositions statutaires communes applicables aux enseignants-chercheurs et portant statut particulier du corps des professeurs des universités et du corps des maîtres de conférences. Repéré à https://www.legifrance.gouv.fr/eli/ decret/2017/5/9/MENH1704494D/jo.

Ménard, L. (2017). Les effets de la formation à l'enseignement et de l'accompagnement des nouveaux professeurs d'université sur leurs pratiques. Dans P. Pelletier et A. Huot (éd.), Construire l'expertise pédagogique et curriculaire en enseignement supérieur. Connaissances, compétences et expériences. Québec : Presses de l'université du Québec.

Morrison, D.E. (1997). Overview of instructional consultation in North America. Dans K.T. Brinko et R.J. Menges (éd.), Practically speaking: a sourcebook for instructional consultants in higher education (p. 121-129). Stillwater, OK : New forums.

Musselin, C. (2008). Vers un marché international de l'enseignement supérieur ? Critique internationale, 39(2), 13-24.

Paivandi, S. (2015). Apprendre à l'université. Bruxelles : De Boeck.

Pastré, P., Mayen, P. et Vergnaud, G. (2006). La didactique professionnelle. Note de synthèse. Revue française de pédagogie, 154, 145-198.

Piaget, J. (1975). L'équilibration des structures cognitives : problème central du développement. Paris :

Presses universitaires de France.

Piot, T. (2008). La construction des compétences pour enseigner. McGill Journal of Education, 43(2), 95-110.

Potocki-Malicet, D. (1997). Les règles de scolarité dans l'Université : importance et rôle des règles et des pratiques locales. Sociétés contemporaines, 28, 57-76.

Rege Colet, N. et Berthiaume, D. (2009). Être ou savoir ? Identité professionnelle et savoirs professionnels chez les enseignants universitaires. Dans R. Hofstetter et B. Schneuwly (éd.), Savoirs en (trans)formation. Au cœur des professions de l'enseignement et de la formation (p. 137-162). Bruxelles : De Boeck université.

Rege Colet, N., McAlpine, L., Fanghanel, J. et Weston, C. (2011). Le concept de Scholarship of Teaching and Learning. Recherche et Formation, 67, 91-104. 
Remmik, M., Karm, M., Haamer, A. et Lepp, L. (2011). Early-career academics' learning in academic communities. International Journal for Academic Development, 16(3), 187-199.

Rey, B. (2009). Autour des mots. « Compétence » et « compétence professionnelle ». Recherche et Formation, 60, 103-116.

Rey, B. (2014). La notion de compétence en éducation et formation. Enjeux et problèmes. Bruxelles: De Boeck

Schön, D. (1983). The reflective practitioner: how professionals think in action. New York, NY: Basic Books.

Stes, A. et Van Petegem, P. (2011). La formation pédagogique des professeurs dans l'enseignement supérieur. Une étude d'impact. Recherche et Formation, 67, 15-30.

Tas (s.d.). Dans Ortolang. Centre National de Ressources Textuelles et Linguistiques. Repéré à http:// www.cnrtl.fr/definition/tas.

Theall, M. et Arreola, R. A. (2006). The Meta-profession of Teaching. Higher Education Advocate, 22(5), 5-8.

Tremblay, N. (1986). Apprendre en situation d'autodidaxie : une étude des besoins des apprenants et des compétences des intervenants. Montréal : Presses Universitaires de Montréal.

Verrier, C. (1999). Autodidaxie et autodidactes. L'infini des possibles. Paris : Anthropos.

Wenger, É. (1998). Communities of Practice: Learning, Meaning, and Identity. Cambridge : University Press.

Wenger, É. (2005). La théorie des communautés de pratique : Apprentissage, sens et identité. Québec : Presses de l'Université Laval.

\section{NOTES}

1. Traduction personnelle de l'expression « on-the-job learning » utilisée par Knight et al. (2006).

\section{RÉSUMÉS}

Le métier d'enseignant universitaire s'apprend le plus souvent "sur le tas » (Knight, Tait et Yorke, 2006). Que recouvre précisément cet apprentissage "sur le tas»? Cet article vise à répondre à cette question en identifiant les pratiques d'apprentissage que les universitaires novices mettent en œuvre pour construire leurs compétences en enseignement. Dans ce but, en 2016, un questionnaire administré à 376 maîtres de conférences en France a permis de relever que les pratiques d'apprentissage de ces derniers sont éclectiques et tendanciellement nonstructurées. Ces pratiques pourraient traduire une aspiration à l'autonomie, c'est-à-dire la capacité à prendre en charge la responsabilité de la construction de leurs compétences d'enseignement leur apprentissage à l'enseignement. À l'heure où la formation pédagogique des enseignants-chercheurs de l'université en France relève d'une obligation statutaire (Le Journal officiel de la République française, 2017), les résultats de cette recherche engagent à une 
réflexion sur une formation pédagogique des universitaires qui prendrait en compte la diversité des pratiques et l'aspiration des novices à l'autonomie.

How to teach in academia is most often learnt on-the-job (Knight, Tait et Yorke, 2006). But what does the phrase "on-the-job learning" genuinely mean in this case? This article aims to identify which learning practices novice academics make use of to acquire their teaching competences in France. In 2016 a survey carried out among 376 novice lecturers showed that novices' practices are eclectic and mainly informal. These practices are the reflection of the academic novices' wish to be autonomous. While public authorities have been developing formal training structures targeted at all newly-hired academics (Le Journal officiel de la République française, 2017), this research suggests that the variety of practices should be taken into account.

\section{INDEX}

Mots-clés : enseignants universitaires novices, construction des compétences, modèles d'apprentissage, formation à l'enseignement, pédagogie universitaire

\section{AUTEUR}

\section{SACHA KIFFER}

Université de Rennes, Rennes

sacha.kiffer@univ-rennes2.fr 